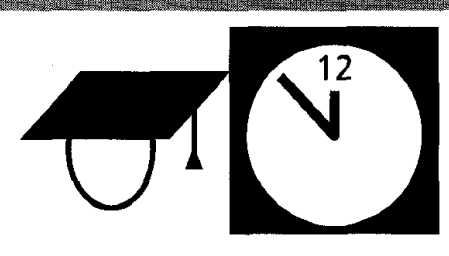

\title{
Satellite ATM Network Architectures: An Overview
}

\author{
Chai-Keong Toh, Georgia Institute of Technology \\ Victor O. K. Li, University of Hong Kong
}

\begin{abstract}
A satellite ATM network has been envisioned as the next information super-skyway. There has been a migration from traditional bent pipe satellites to onboard processing) ATM satellites. The current congestion in the Internet has motivated the use of alternate paths - using satellites to support global Internet transport and access. This article presents the various system and protocol layer architectures of SATM networks.
\end{abstract}

\section{$T$}

he global information infrastructure (GII) of the United States has included satellite asynchronous transfer mode (SATM) as part of the communications infrastructure to support global mobile communications, information transport and access. The Telecommunications Industry Association (TIA) has recently formed an SATM group to study the various technical issues (e.g., reference models, media access, and mobility management) related to SATM. In addition, the ATM Forum Wireless ATM Group has established liaison with TIA to initiate standardization efforts on SATM [1]. This article reflects the increased interest expressed by industry, academia, and regulatory bodies in SATM network architectures.

Current satellite network configurations comprise mainly ground, user, and space segments.

The ground segment consists of gateway Earth stations (GESs), a network control center (NCC), and operation control centers (OCCs). Satellites communicate with fixed and mobile users via satellite-user links and gateway sta-

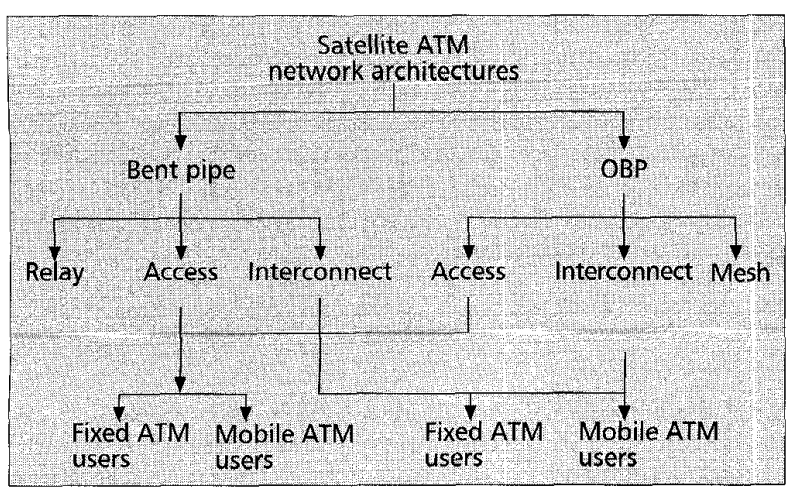

Figure 1. Overview of various bent-pipe and OBP SATM network architectures. tions. Location tracking of mobile terminals is performed at GESs. Most of the GESs are connected to mobile switching centers of the land mobile network. The ground NCC provides functions such as mobile registration, verification, billing, network database distribution, and network resource allocation and management. OCCs, on the other hand, are responsible for satellite orbital tracking, ranging, and control. Processed information from OCCs is then distributed to GESs in order to support location tracking and handoffs. The user segment relates to a fixed, handheld, or vehicle-mounted unit requiring global integrated services (data, voice, and video). A dual-mode mobile user terminal can have access to both satellite and terrestrial cellular/wireless networks. Finally, the space segment consists of low $/ \mathrm{middle} /$ geostationary Earth orbit (LEO/MEO/GEO $)^{1}$ satellites with or without intersatellite links (ISLs) and OBP capability.

Satellites are now commonly termed based on their distance from and spatial relationship with the earth. A GEO satellite orbits at an altitude of $22,300 \mathrm{mi}$ above the earth, providing substantially wide coverage. An example application of GEO service is mobile car phone systems. LEOs and MEOs orbit at 500-7000 mi in altitude; compared to GEOs, LEOs are nearer to the Earth, making smaller handheld devices possible. However, because LEOs/MEOs yield smaller coverage areas, a constellation of satellites is needed to provide coverage of the Earth. The Global Positioning System (GPS), for example, uses 24 LEOs satellites to provide position and location services for portable and vehicle-mounted GPS devices.

Traditional satellites, especially GEOs, mainly act as

${ }^{1}$ Although the terms LEO, MEO, and GEO are used throughout this article, it is recognized by the International Telecommunication Union (ITU) that geostationary orbit is addressed by the term GSO, not GEO; and both $M E O / L E O$ are addressed by the term NGSO (non-geostationary orbit). 


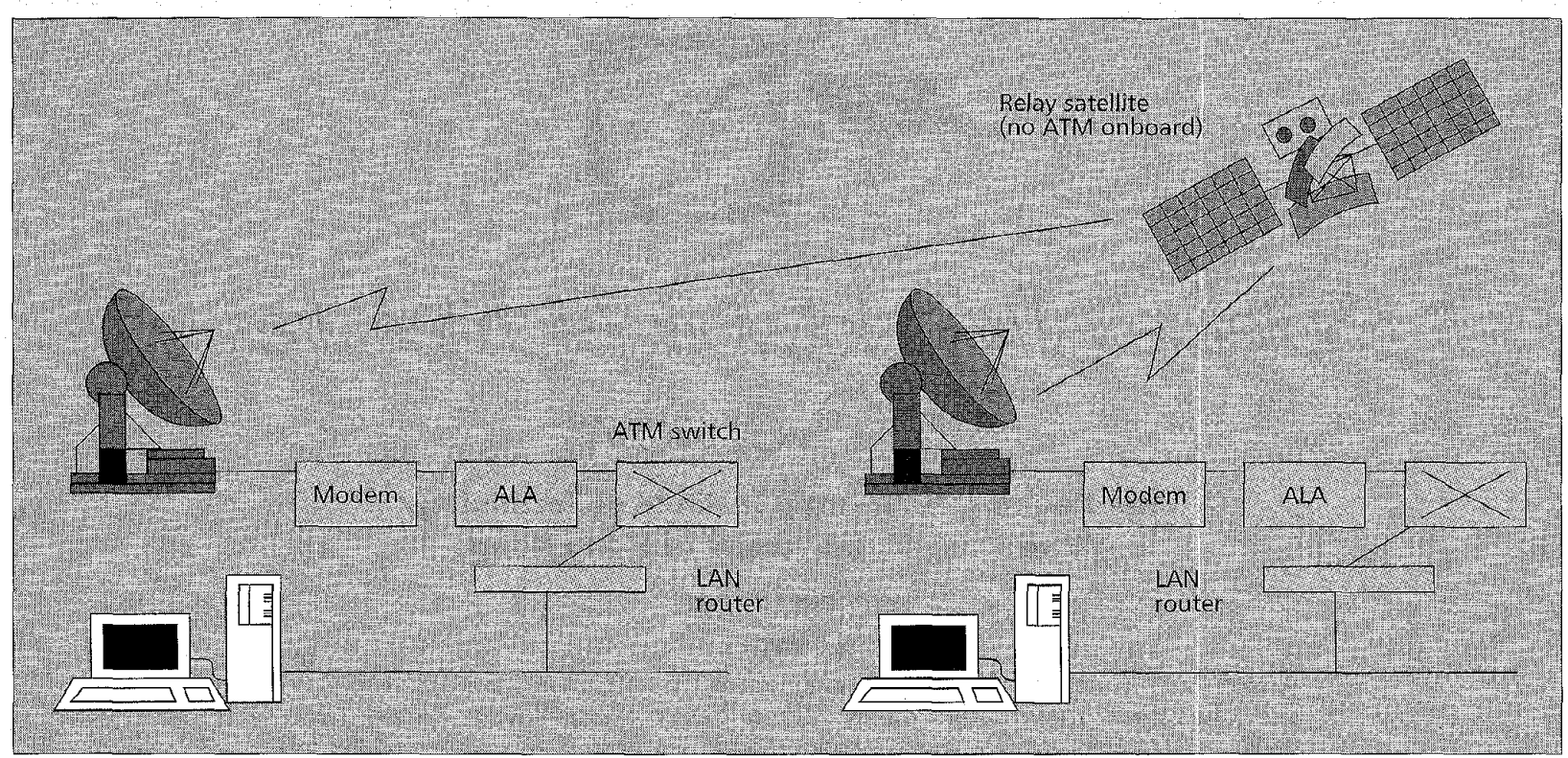

Figure 2. Current SATM relay architecture.

relays, providing a communication pipe linking two or more ground-based communication points, hence, they are termed bent-pipe satellites. These satellites provide point-to-point and also point-to-multipoint linkage. Beam multiplexing is employed at the satellite so that a higher efficiency of link utilization can be achieved. However, with the advent of multimedia communications and high-speed networks, the performance and functionality associated with existing satellites has to be improved in order to support new applications. Several projects, ${ }^{2}$ experiments, and field trials (e.g. ACTS, ${ }^{3}$ NICE, ${ }^{4}$ VANTAGE, ${ }^{5}$ WISDOM ${ }^{6}$ ) were conducted in the United States and Europe to evaluate issues associated with sending ATM cells over satellite and using the satellite to interconnect between two regionally separated ATM networks. The idea of "implanting" ATM switching technology onboard the satellite gives rise to OBP ATM satellites, such as Cyberstar [2], Astrolink [3], Spaceway [4], and Skyway [5]. The Skyway network, comprising 12 continent-wide coverage satellites, is an example of a commercial gigabit OBP satellite system. This article focuses its discussions on these two broad categories of SATM architecture. Based on the types of functionality (relay, access, or interconnect), users (fixed or mobile), and network platforms (fixed or

${ }^{2} A$ summary of some ongoing SATM projects is provided in [13].

${ }^{3}$ The Advanced Communications Technologies and Services (ACTS) program is the European Commission's major program for supporting precompetitive research and technological development in telecommunications, in the period 1995-1998.

${ }^{4}$ NICE: National Hosts InterConnection Experiment - A project related to the interconnect of national hosts by means of terrestrial and satellite ATM links.

5 VANTAGE: VSAT ATM Network Trials for Applications Groups across Europe - A project to demonstrate user access to ATM networks from very small aperture terminals (VSATS) at lower bit rates.

6 WISDOM: WIdeband Satellite Demonstration of Multimedia - A project involving the building of an end-to-end system demonstrator using $a$ satellite with onboard ATM switching capabilities.

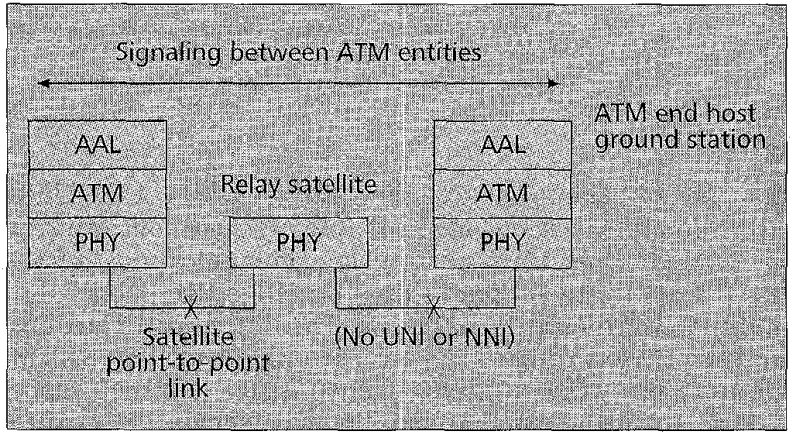

Figure 3. Protocol layer model for SATM relay architecture.

mobile), several SATM network architectures may be realized, as illustrated by Fig. 1. We shall discuss some of these existing and emerging SATM architectures.

Several existing articles on SATM have been devoted to physical, data link, media access, quality of service (QoS), and routing issues [6-9]. However, in this article we focus strictly on architectural issues for bent-pipe and OBP SATM networks. This article is an extension of contributions to TIA and ITU [10-12] and it is organized as follows: the second section presents the system and protocol layer architectures for bent-pipe relay, access and interconnection SATM networks. Issues related to signaling interfaces and protocollayer functions will also be discussed in this section. The third section provides a similar discussion but for OBP SATM architectures. A summary of the various architectures is provided at the end of the second and third sections, and we conclude in the fourth section.

\section{Bent-Pipe SATM Network Architectures SATM Relay Architecture}

Satellite as a relay is the simplest SATM architecture, where the satellite link is treated as a communication pipe to replace a terrestrial link and to relay ATM traffic from one remote ATM user to another. The satellite, however, does not switch ATM cells at the ATM layer, that is, does no vir- 
tual path (VP) or virtual channel (VC) switching. Furthermore, satellite links are static; therefore, there is no need for a media access layer to support multiple access, bandwidth negotiation, or handoff. This architecture is currently implemented using GEO satellites. As shown in Fig. 2, since the satellite link is regarded as an ATM link, ATM cells cannot be fed directly to the satellite since some rate adaptation and cell preprocessing are required. Because the quality of satellite links is dependent on weather conditions, burst errors can severely affect ATM QoS parameters [14], especially cell loss ratio (CLR) and cell discard probability. Several error control schemes (e.g., bit interleaving) have been proposed to resolve this problem. The ATM link accelerator (ALA) [15] provides ReedSolomon-based forward error correction (FEC) and interleaving, thereby providing fiber-link-like quality over noisy satellite links. The protocol layering architecture shown in Fig. 3 reveals that there is no ATM-related signaling between the user terminal and the satellite. Signaling will only occur between the two ground ATM switches during call setup and teardown. Therefore, this architecture can be deployed in most existing satellites since no ATM switching and signaling functions are required onboard the satellite.

\section{SATM Network Access Architectures}

Access between Fixed ATM Users - This architecture provides ATM network access to end devices. ATM connections can be established between an ATM uscr and an ATM network or between two ATM users. The interface between an ATM user and an SATM access point is a public or private user-network interface (UNI), depending on the type of ATM network at the other end of the connection. As shown by Fig. 4, connections between ATM end devices and the fixed access point (satellite terminal or Earth station) can be wired or wireless. The access point at the ATM network side is a GES. The GES acts as a multi-

plexer and is connected to the ATM network by a network-network interface (NNI) such as the ATM Forum's private NNI (PNNI) in the case of a private ATM network. If the connection is with a public ATM network, this interface is a public UNI. This architecture is characterized by a large number of low-cost small-size user terminals and a few GESs. Mobility support is not provided in this architecture because users are static. Reference point S1.1.A is a standard ATM UNI including the user, control, and management planes. Reference point S1.1.B is the common air interface between the user terminals and GESs. Reference point S1.1.C is a public UNI if the connection is with a public ATM network, or a PNNI if the connection is with a private ATM network.

The protocol reference model for SATM access is shown in Fig. 5. At the end user, the native ATM protocol stack is present. User information is segmented via the ATM adaptation layer (AAL) into fixed-size ATM cells before being transmitted over the wired ATM interface. A UNI signaling entity is required to allow the user to establish

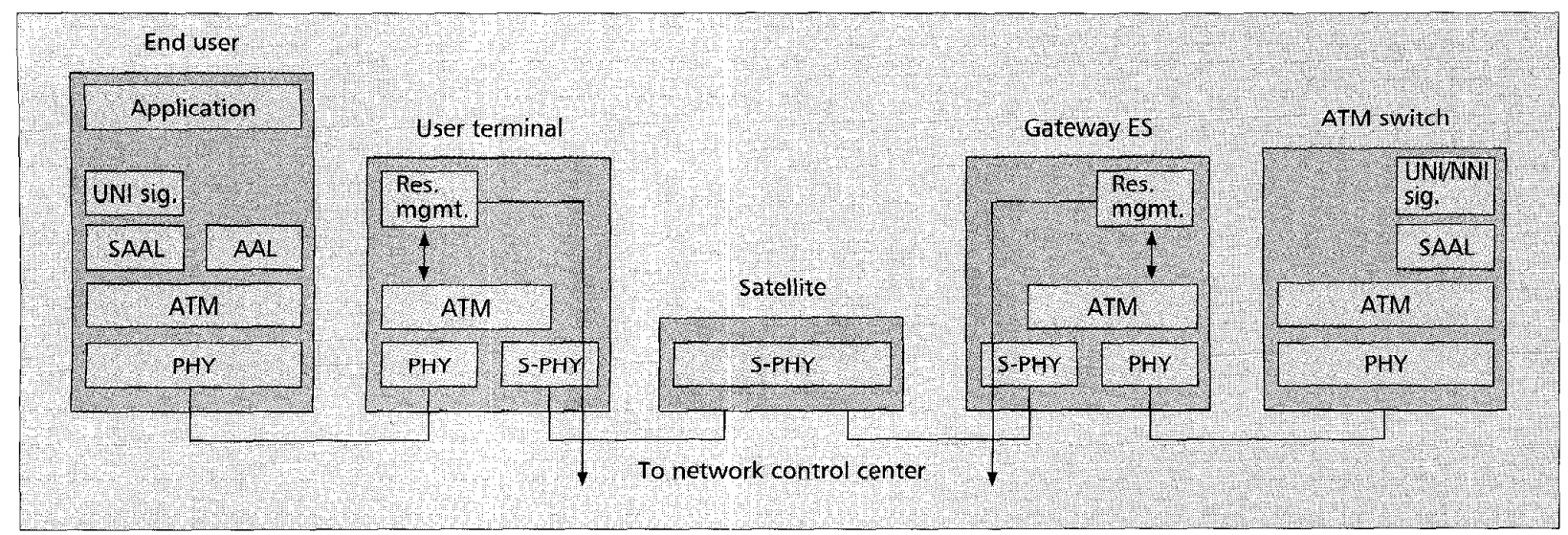

Figure 5. SATM access bent-pipe protocol layer architecture (fixed users). 


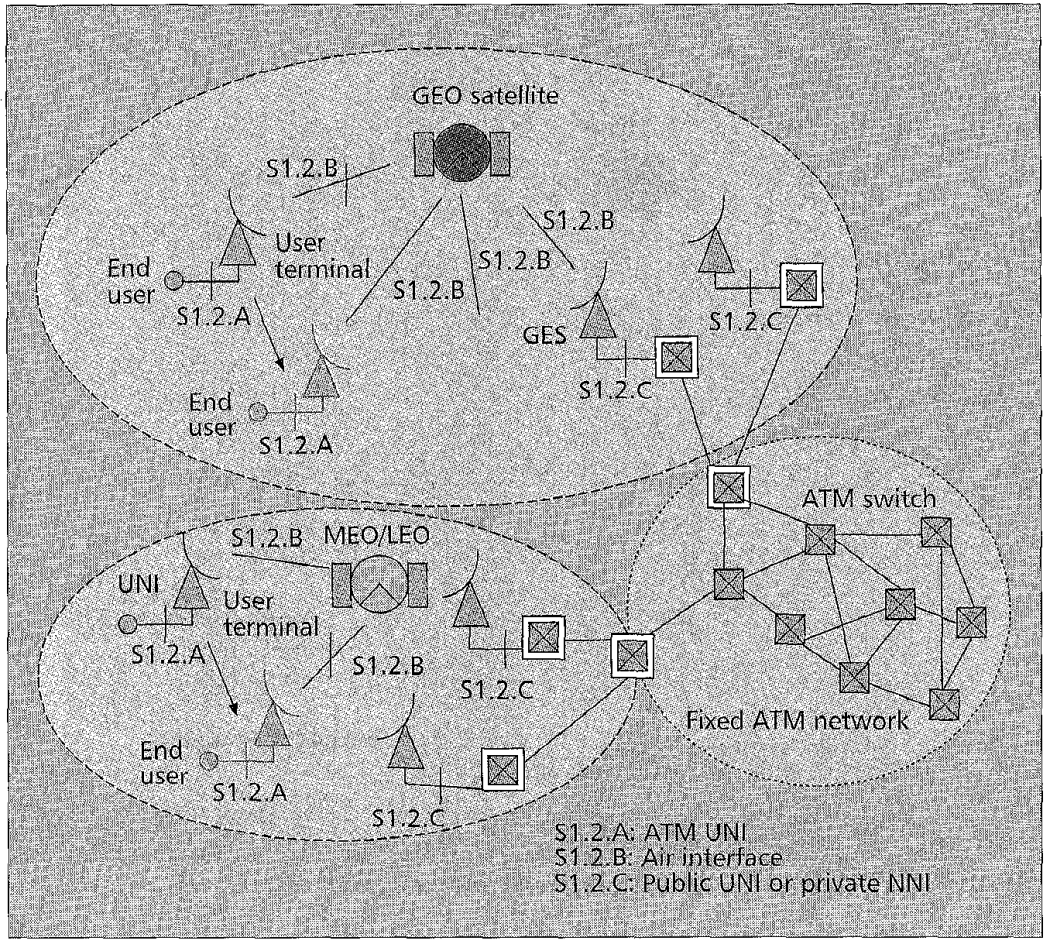

Figure 6. SATM access bent-pipe protocol layer architecture (mobile users). the end user except that it has a UNI/NNI entity to allow it to interact with the end user (via the UNI) and the network (via the NNI) to support connection establishment.

Access between Mobile ATM Users Contrary to the earlier architecture, this architecture provides ATM network access to both moving and portable end devices. With moving devices, this architecture has to support location management (i.e., authentication, registration, paging, roaming, routing) and handoffs of mobile connections between access points. As shown in Fig. 6, the interface between mobile ATM user equipment and an SATM terminal adapter (i.e., S1.2.A) is a mobility-enhanced UNI (M+UNI) which supports hand-off and location-related signaling. The access point at the ATM network side is a fixed GES. The GES acts as a multiplexer and is connected to the ATM network via a mobility-enabled ATM switch (MES). When a user migrates outside the coverage of an existing satellite into the coverage area of another new satellite, on-going connections will be switched/hand-off via the MES.

connections over the SATM network. Note that this signaling entity corresponds with another private UNI/NNI entity residing at the remote ATM switch, which is connected to the fixed ATM network. Neither the user terminal nor GES is involved in UNI signaling. The user terminal is a device acting as a gateway between the wired ATM station and the satellite; hence, it has both ATM and satellite physical layers. The ATM layer is still required so that cell switching, multiplexing, and interpretation of VCs/VPs can be performed. A resource management (RM) entity is required at the user terminal to negotiate with the NCC for network resources. This RM entity is also present at the GES since it serves as the gateway node for servers residing in the fixed ATM network to communicate with remote end users. Again, the ATM layer is present at the GES for similar cell-related functions. Finally, the gateway ATM switch possesses the same protocol stack structure as
Reference point $\mathrm{S} 1.2$. B is the common air interface between the mobile terminal and the GESs. This interface requires a media access control (MAC) protocol since multiple user terminals may be trying to access the satellite channel. Several MAC schemes have been proposed and studied, such as Time Division Multiple Access (TDMA) [6], Dynamic TDMA, Multiple Frequency TDMA [9]: Reference point S1.2. C is identical to S1.2.A. since they are all referring to the same M+UNI. The protocol reference model for SATM access between mobile ATM users/networks is shown in Fig. 7. Similar to the earlier architecture, RM functions are implemented at the user terminal and the GES. ATM UNI signaling messages are used for call control. These messages are transported transparently over the satellite and are used by the RM entity to allocate/deallocate required satellite capacity during call setup. GESs use internal signaling to request channel capacity from the NCC or to release capaci-

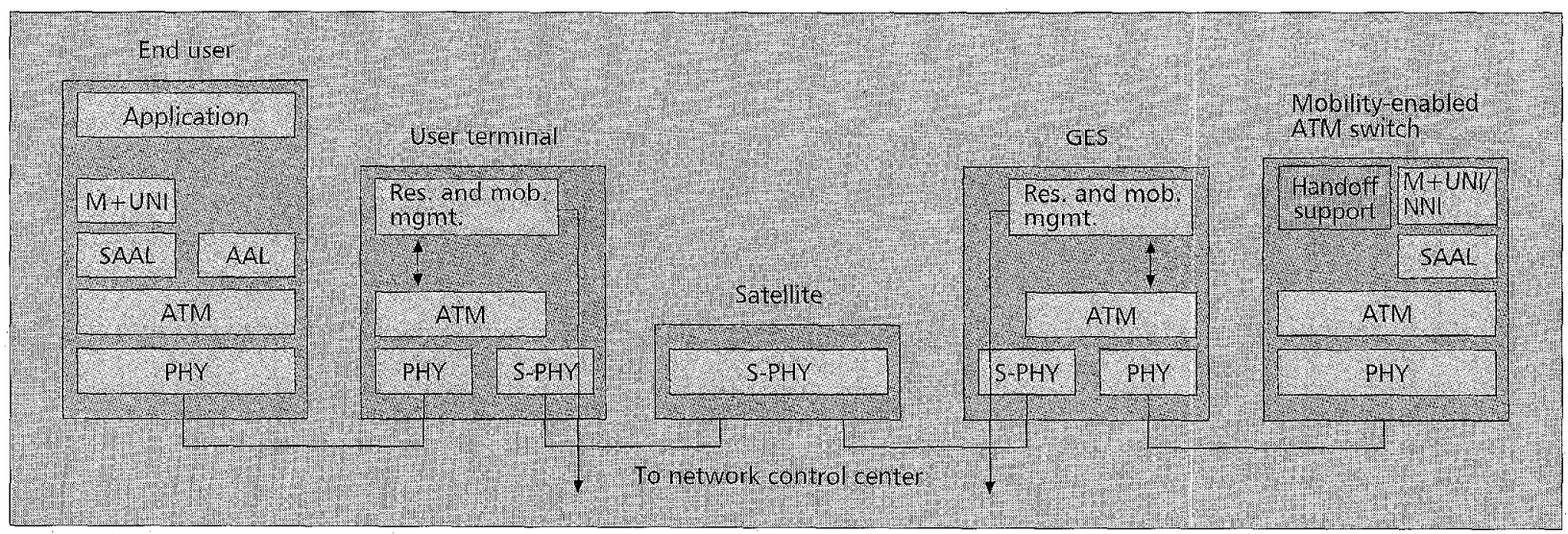

Figure 7. SATM access bent-pipe protocol layer architecure (mobile users). 
ty based on this information. Mobilityrelated signaling functions are present at the user terminal and the GES to support location management and handoffs. Since the end user will always be attached to the same user terminal, there is no mobility between the end user and the user terminal. All mobility management functions can be handled by M+UNI signaling between the user terminal and the GES. The MES connected to the GES, however, implements ATM-layer handoff [16] functions, such as rerouting ATM traffic from the current GES to the newly associated GES currently servicing the migrated end user. As before, the satellite acts as a relay pipe with no signaling and cell switching functions.

\section{SATM Interconnection Architectures}

Interconnection between Fixed ATM Networks - Besides cell relay and network access services, the ability to provide interconnections of ATM networks via satellite [17] is also important. The interconnection can be between two or more fixed ATM networks or mobile ATM networks. By "mobile ATM networks" we refer to private ATM networks with satellite connectivity housed in an airplane, a multimedia truck/van, or even a mobile home. This subsection describes the SATM architecture for interconnecting fixed ATM networks. As shown in Fig. 8, the interfaces between the SATM access points and the terrestrial ATM networks (i.e., S1.3.A) can be PNNI, broadband intercarrier interface (B-ICI), or a public UNI. If the satellite interconnection is for two private ATM networks, the interface between the access points and the ATM network is a PNNI. For interconnection between two public ATM networks, however, this interface becomes a B-ICI. Since the satellite is used solely for interconnection of fixed ATM networks, no mobility support is provided in this architecture; this explains the absence of MESs. Reference point S1.3.B is the common air interface among the GESs. Figure 8 also shows that an SATM connection may involve several GESs and satellites.

The protocol reference model for SATM interconnection is shown in Fig. 9. Since the satellite serves as the interconnection relay between two fixed ATM networks, the protocol structure does not contain any handoff entity or mobilityrelated signaling entity. Both ends of the ATM switches

implement basic ATM physical- and ATM-layer functions. There is no AAL since these are intermediate nodes, not end systems. The SAAL provides reliable transport of signaling messages (e.g. ITU Q.2931 or ATM Forum UNI) between peer Q.2931/ATM Forum UNI entities. GESs in the route path interact with the NCC via internal signaling to negotiate for network resources. Finally, as before, the satellite acts as a relay pipe with no cell switching/multiplexing functions onboard.

Interconnection between Mobile ATM Networks - This emerging architecture provides high-speed interconnections for:

- A mobile and a fixed ATM network

- Between two mobile ATM networks

An implementation example is the rapidly deployable radio network (RDRN) [18]. A mobile ATM switch (this is not the MES, but refers to a switch that resides in a mobile vehicle/device) can be used to provide network access for a group of ATM end users via the bent-pipe satellite. As shown in Fig. 10, interface S.1.4A is a mobility-enhanced NNI $(\mathrm{M}+\mathrm{NNI})$ since it is concerned with signaling between MESs.

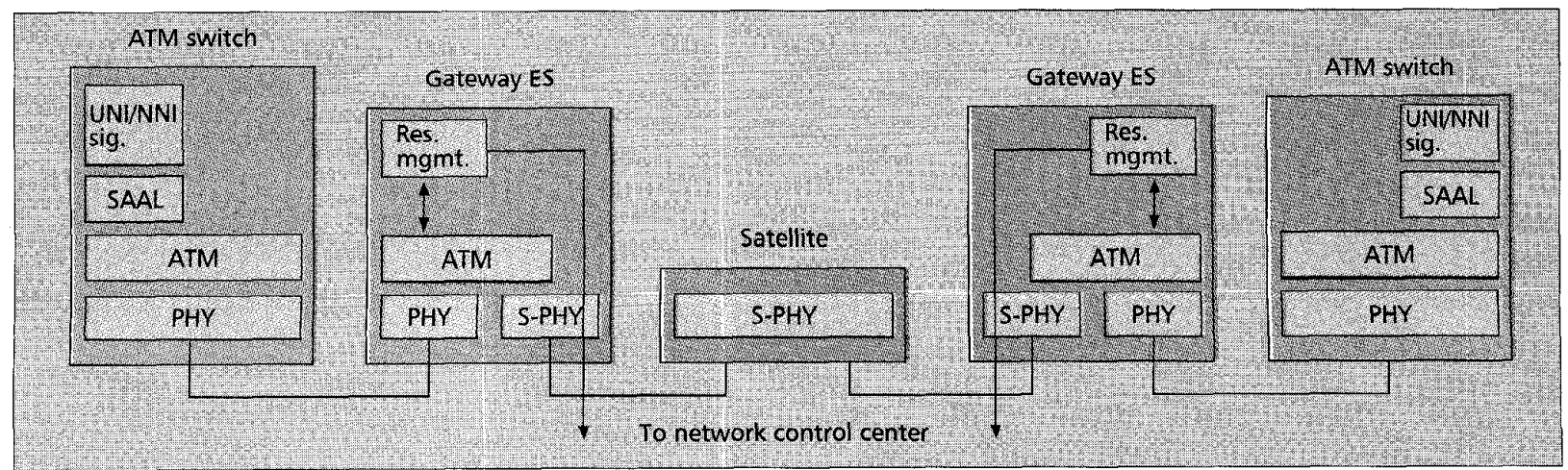

Figure 9. Bent-pipe SATM interconnection protocol layer architecture (fixed networks). 


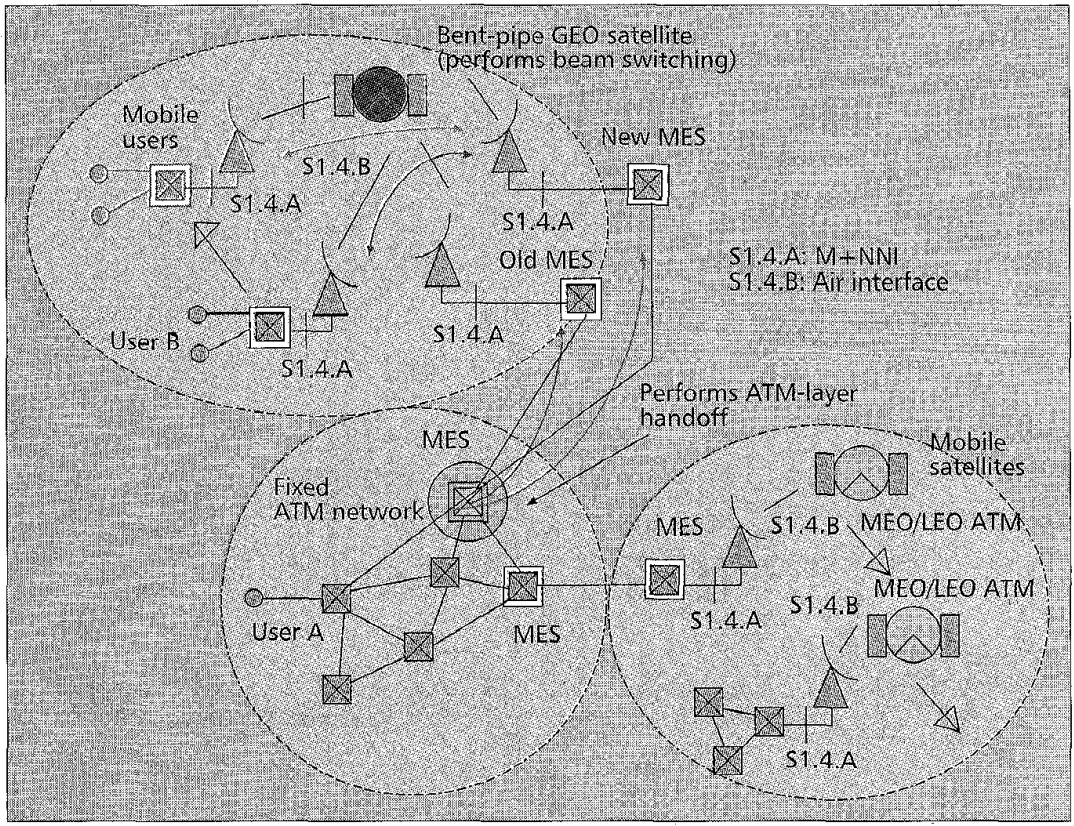

Figure 10. Bent-pipe SATM interconnection architecture (mobile networks).

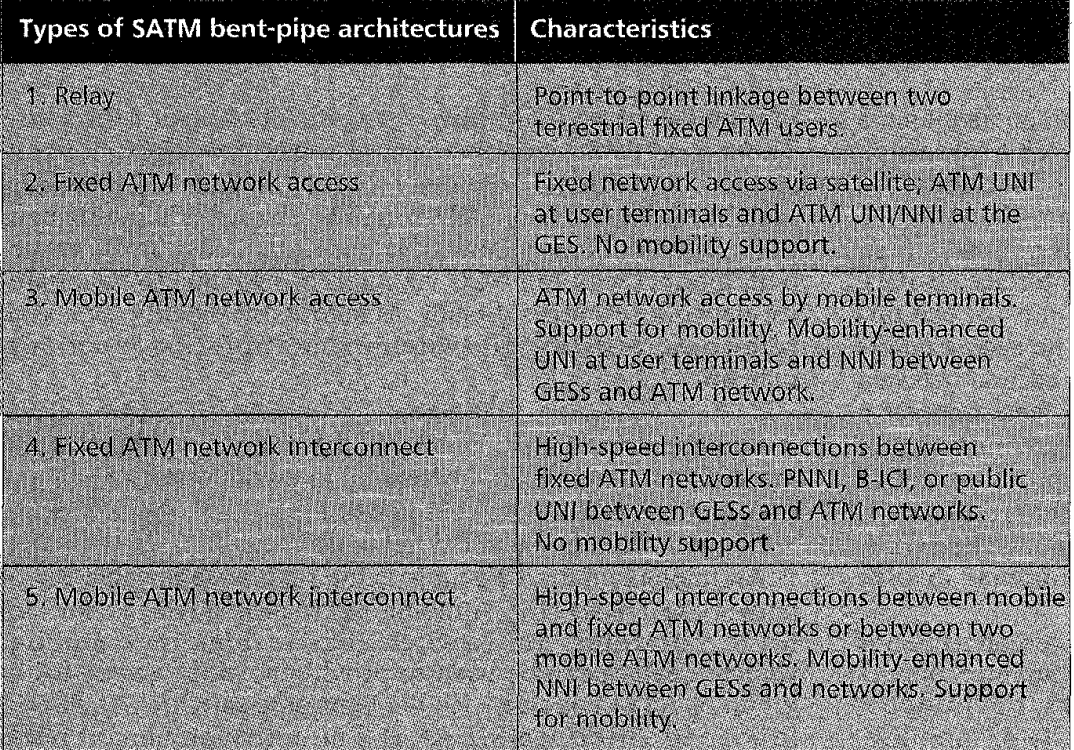

Table 1. Summary of bent-pipe SATM network architectures.
Reference point S1.4.B is the common air interface between the mobile/fixed Earth stations. Two scenarios are highlighted in this architecture. The first scenario is when a portion of the network is mobile and the satellite type (GEO, MEO, or LEO) is unspecified. The second scenario is when networks are fixed but the satellite is mobile (i.e., nonGEO). A typical application of this network is to provide connectivity to a group of users on a mobile platform such as a ship, an airliner, or a train. The applicable data rate depends on the speed of the mobile ATM network and the channel quality of the transportation medium. For airborne platforms such as airplanes, the channel quality is higher than land-based platforms since rain fades are usually not observed, but the high speed of the plane can impose an upper limit on feasible data rates. On the other hand, for land-mobile networks such as warships or vessels, the channel quality is poorer due to rain fades, and this governs the achievable data rate. Since ATM networks are mobile, mobility support must be provided for handoffs and location management. In addition, as LEO satellites move about their orbits, their points of access with the GES change over time, requiring handoff [19] of all existing connections supported by the LEO satellite.

The protocol reference model shown in Fig. 11 reveals that mobility-related functions are required at the MES and GES. The handoff function (VC rerouting) is implemented at the MES, while location management functions (i.e., authentication, registration, paging, roaming, routing) are performed by the GES. The NCC stores and maintains location information of mobile users. GESs interact with the ground-based NCC to negotiate for network resources. Since the satellite is acting purely as a repeater, call control and resource management functions must be performed

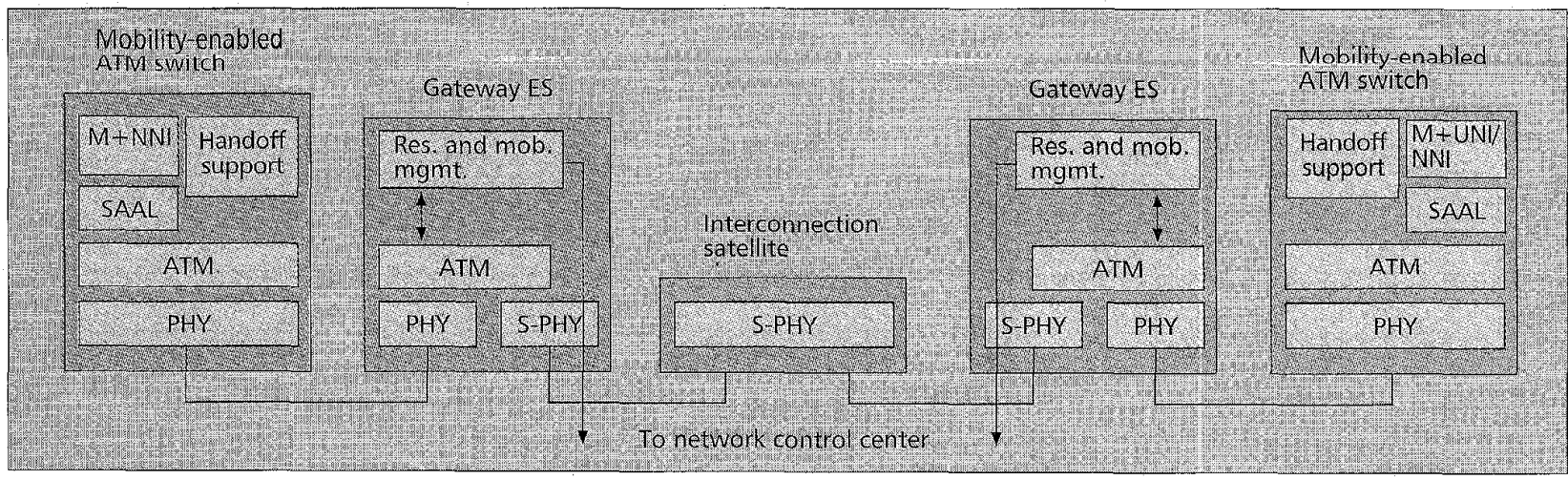

Figure 11. Bent-pipe SATM interconnection protocol layer architecture (mobile networks). 
centrally at the ground NCC. Unlike the earlier SATM network architectures, the mobile ATM switch may now move from the coverage area of one satellite (or beam) to another satellite (or beam). In this case, the ongoing connections between the mobile ATM switch and the old terrestrial MES will have to be handed off to the new terrestrial MES. This will require both a satellite beam handoff (from the old to the new GES) and an ATM-layer handoff at the MES, as illustrated in Fig. 10 (handoff of an SATM connection between users A and B). Therefore, this architecture will require mobility enhancements to the NNI. Table 1 summarizes the characteristics associated with each of the above-mentioned SATM architectures. The relay and fixed ATM network access architectures exist, while the mobile ATM network access architecture is evolving. Both the fixed and mobile ATM network interconnection architectures are part of existing SATM projects.

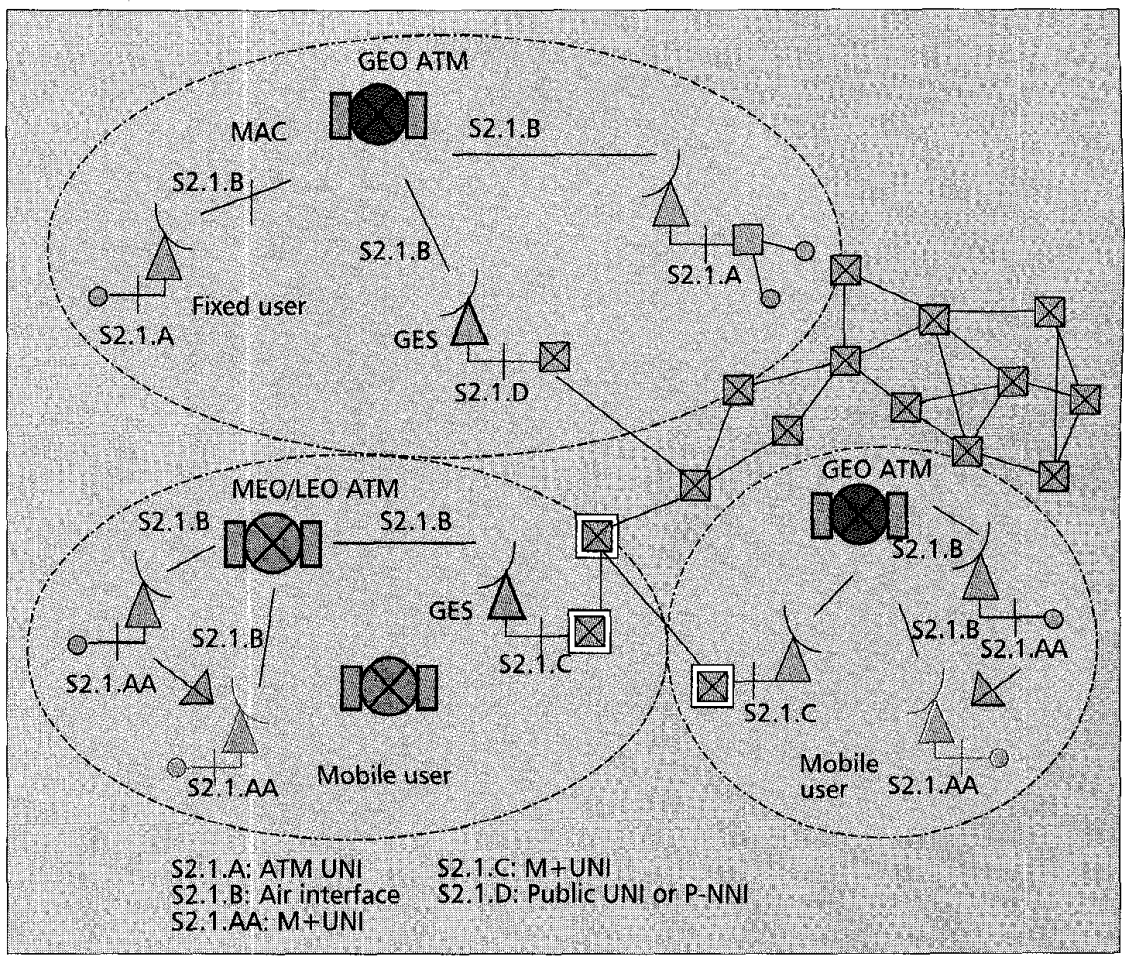

Figure 12. OBP SATM access network architecture (fixed and mobile users' access).

\section{Onboard Processing SATM Architectures}

To achieve unrestricted connectivity, and smaller and cheaper user terminals with lower transmission delay, the ability to switch individual data streams onboard the satellite is desired. This OBP concept was developed by a European and Canadian industrial consortium under the leadership of the European Space Agency [20]. OBP functions include baseband switching and regeneration. Baseband switching concerns the flexible use of channel exchange, regeneration employing FEC techniques on both up- and downlinks so that link margins can be improved [21]. Depending on usage scenarios and system setup, we have identified four possible OBP SATM architectures; each is discussed below. Most of these network architectures are in the design stage, and some are envisioned for future deployment. OBP switch

architectures have also brought about other issues (fault tolerance, switching delays, payload complexity, etc.); these are discussed in [22].

\section{OBP SATM Access Architecture (Fixed/Mobile Users)}

Compared to the corresponding architecture in the bentpipe scenario, the distinction here is that ATM switching is provided onboard the satellite. Users send ATM cells to the satellite (be it GEO, MEO, or LEO), where these cells are multiplexed and switched at high speed (via VP and/or VC routing) to the appropriate output modulator. From there, a downlink connection to a ground GES (which is connected to a fixed terrestrial ATM network) is provided. A medium access control (MAC) protocol is required at the satellite to support multiple access (uplink) by several user terminals. The SATM link is being used to connect remote ATM hosts to a terrestrial ATM network. A multi-

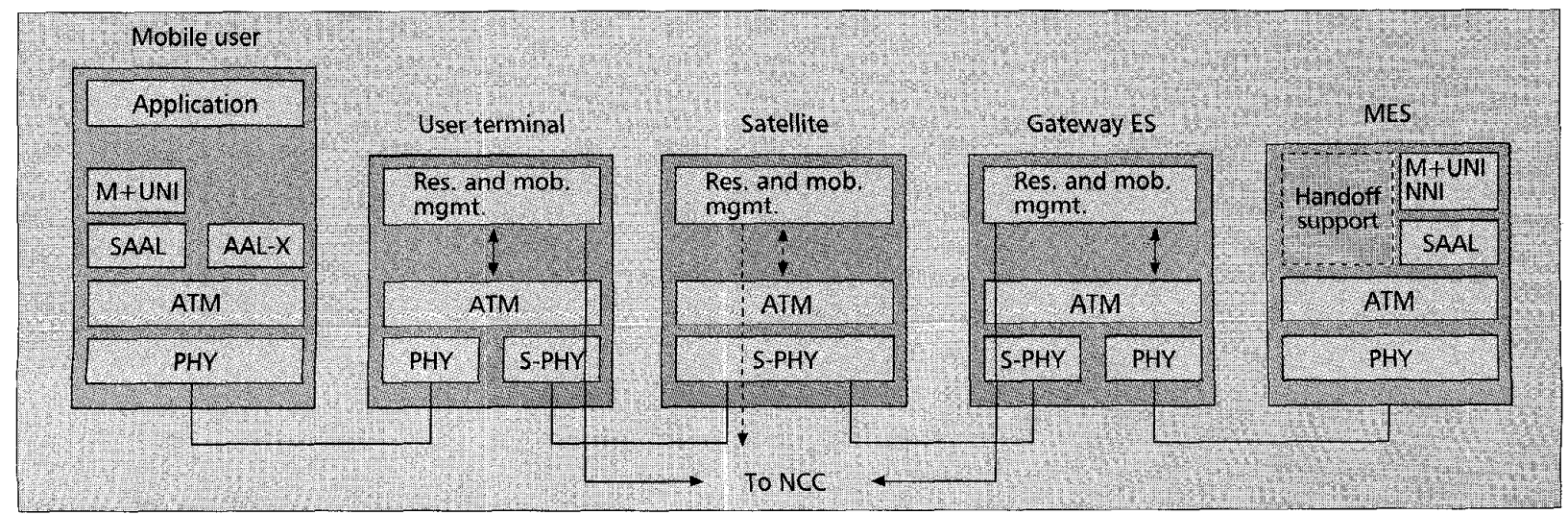

Figure 13. OBP SATM access protocol layer architecture (mobile user). 


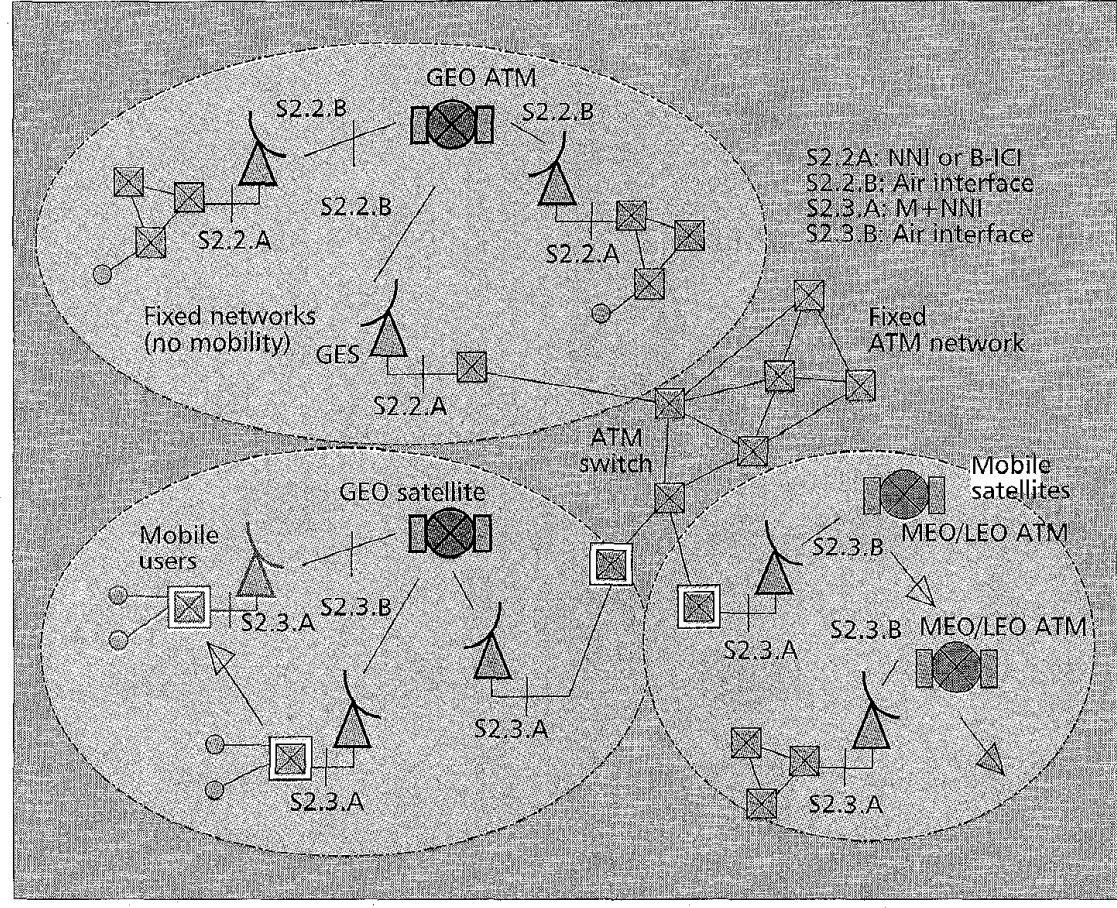

Figure 14. OBP SATM interconnection network architecture.

point-to-point and point-to-multipoint configuration is used for uplink and downlink communications respectively. This architecture is particularly suitable for a scenario where multimedia application servers reside in the terrestrial ATM network, and remote ground ATM stations (e.g., home users) request services from these servers via the satellite. Since ATM supports multicast, the onboard ATM multicast fabric will duplicate ATM cells and route them to different destinations.

As shown in Fig. 12, reference point S2.1.A is a standard ATM UNI. Reference point S2.1.B is the common air interface between user terminals and the satellite. S2.1.C and S2.1.SAA are the M+UNI interface between GES/user terminal and the MES, respectively. Reference point S2.1.D is a public UNI if the connection is with a public ATM network or an NNI if the connection is with a private ATM network. The protocol reference model for OBP SATM access is shown in Fig. 13. The resource management (RM) function implemented at the user terminal, GES, or onboard the
ATM satellite is invoked by ATM UNI signaling messages for the purpose of resource allocation and control. This function can be optionally implemented onboard the satellite, but this is likely to increase satellite complexity. If implemented, the user terminal simply transmits UNI signaling messages to the onboard ATM switch, and the RM entity onboard uses the received information to allocate/deallocate satellite capacity and channel resources. An alternate approach is to implement MAC functions in the groundbased NCC; channel access decisions are then passed back to user terminals via the satellite so that they can access the channels in a preallocated contentionless manner. This methodology trades off delays with the processing complexity required at the satellite. If RM functions are performed at the OBP ATM satellite, coordination with the NCC is still required in order to support QoS end-to-end. This is illustrated by the dotted line in Fig. 13. For SATM connections that are concerned with fixed ATM users, no mobility support is necessary. However, for mobile users handoffs are supported through the MES or collaboratively with the GEO/MEO/LEO ATM satellites. Call control and routing decisions are made by the NCC. The functions of PHY, S-PHY, SAAL, ATM layer, and UNI/NNI signaling layers are similar to those explained earlier.

\section{OBP SATM Interconnection Architecture (Fixed/Mobile Networks)}

Similar to the bent-pipe interconnection architecture, two scenarios exist for OBP satellites:

- Providing interconnection between fixed ATM networks

- Mobile ATM networks

One major difference, however, is that the satellite is now an ATM node (instead of a repeater) interconnecting multiple fixed or mobile ATM networks. This explains why the signaling interfaces (S2.2.A) associated with the satellite

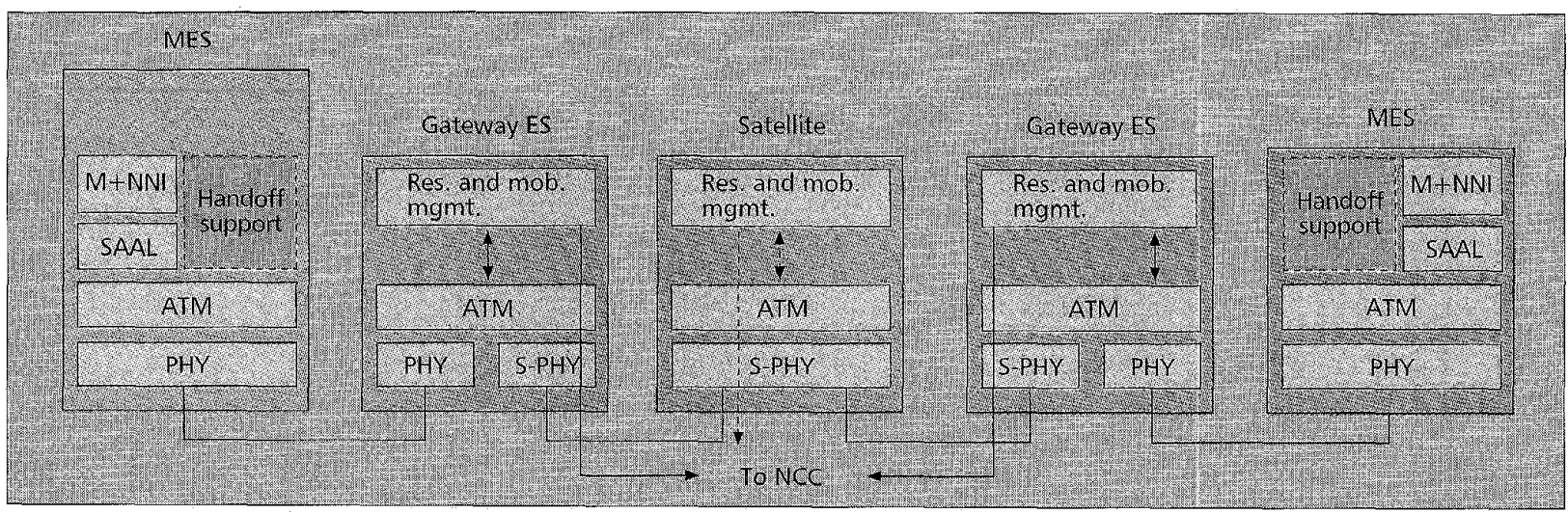

Figure 15. OBP SATM interconnection protocol layer architecture (mobile networks). 
links are of the NNI type. For mobile platforms, this interface (S2.3A) becomes the M+NNI. S2.2B and S2.3B refer to the air interfaces between the Earth stations and the satellite. The satellite can be viewed as a high-capacity ATM switch, switching ATM traffic from one ATM network to another. Hence, the onboard ATM switch must be capable of handling a large volume of traffic. Since the satellite is regarded as an intermediate ATM node, ATM cell-level QoS parameters become an important issue, and this applies to GEO/MEO/LEO satellites. Figure 14 reveals the various scenarios containing fixed users, mobile users, GEO, and non-GEO satellites. Compared to the OBP SATM network access architecture, the satellite is now considered an intermediate ATM node with dedicated incoming links and outgoing links, and a simple MAC function is present at the satellite to support bandwidth control.

To avoid repetitions, a single protocol reference model for OBP SATM interconnection architecture is shown in Fig. 15. For the case of interconnecting between fixed ATM networks, no mobility management entity is required at the GES, unlike those for mobile networks or non-GEO satellites. RM entities are required at the GESs for both fixed and mobile network platforms, and are invoked by ATM NNI signaling messages. The onboard ATM switch has to perform a variety of cell switching, call, and traffic control. In addition, it must also support reservation of network resources during call establishment. Hence, signaling is required between the satellite and the ground NCC to collaboratively support end-to-end QoS requirements, as shown by the dotted line in Fig. 15. For mobile platforms, mobility management (mobile connection, handoff, and location management) functions are required at the ATM switch, GESs, and OBP ATM satellite. Handoffs can be performed by the satellite and/or ground MES. Unlike the corresponding bent-pipe architecture, the satellite performs ATM-layer handoffs.

\section{OBP SATM Full Mesh Architecture}

In this configuration, several onboard ATM satellites form an ATM network in space. The SATM mesh network performs

ATM traffic switching, flow, and congestion control functions, and supports connection setup, teardown, and QoS requirements. These satellites use equivalent NNI or $\mathrm{M}+\mathrm{NNI}$ signaling between themselves, and UNI or M+UNI signaling between them and the GESs. The SATM mesh network can be used to interconnect several fixed or mobile terrestrial ATM networks and also to allow remote ATM stations to access the satellite and establish ATM connections to multiple remote ATM stations. Intersatellite communications [23] are necessary here in order to support ATM connections that go beyond a single satellite hop. Such communications demand routing capability in the satellites. With GEO ATM satellites, the satellite network topology will hardly change over time since they are always pointing to the same positions on the Earth. Hence, static routing may be used where routing tables can be permanently configured prior to the launching of satellites. However, it may be necessary for static routing to be configurable from the ground control station since the ground ATM network topology can change over time (e.g., GESs are relocated). For LEO/MEO ATM satellites, their positions will change over time; hence, dynamic routing [7] is necessary. Lastly, if mobile users are to be supported, handoffs occur when mobile users move beyond the coverage of a satellite. In addition, when a satellite moves

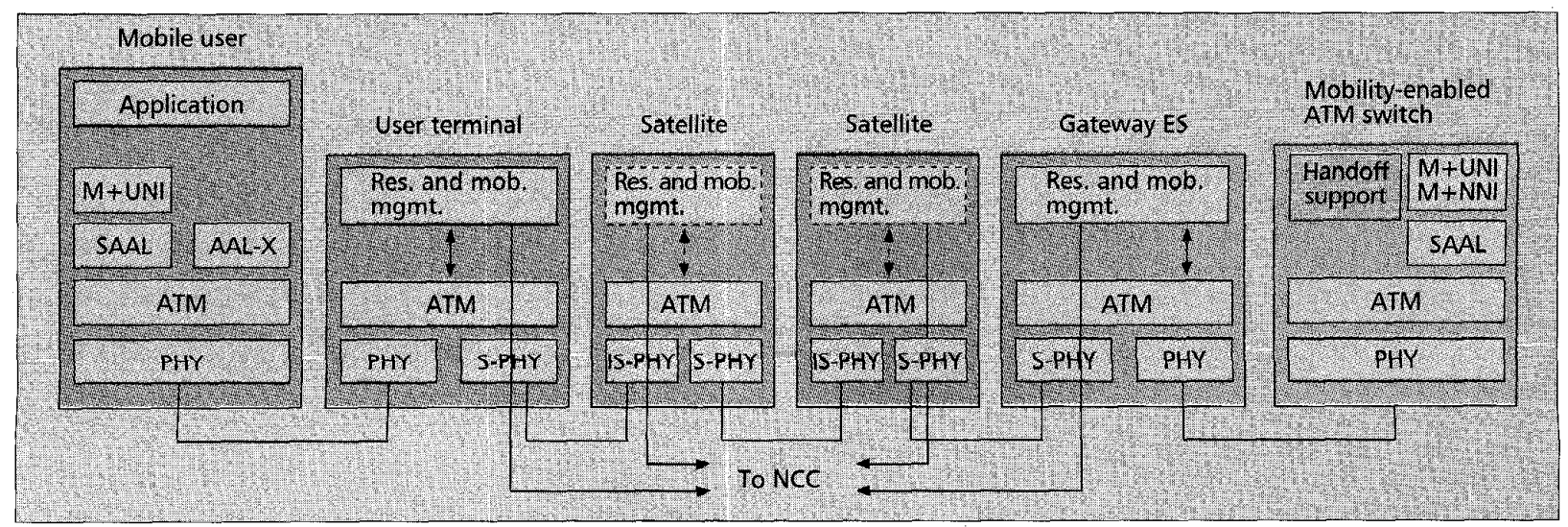

Figure 17. OBP SATM full mesh protocol layer architecture (mobile users). 


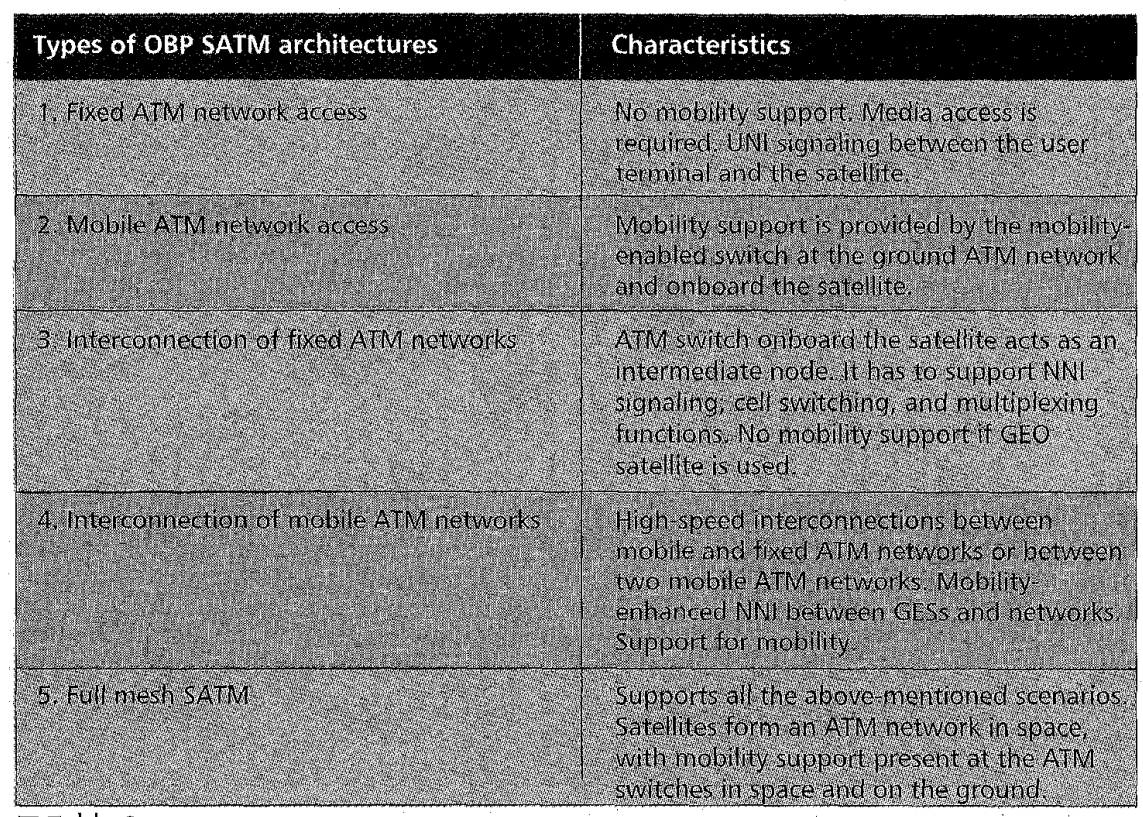

Table 2. Summary of OBP SATM architectures. satellite communicates with GES via the S-PHY, and other satellites via IS-PHY (intersatellite link). Routing and call processing functions can be collaboratively shared by the satellite and NCC. The satellite, however, must be able to support in-plane and cross-plane ISL routing [7] since a connection can involve multiple GESs, satellites, and ground MESs. Summarizing, the above-mentioned OBP ATM satellites may function as:

- An access node (like a wireless base station)

- An interconnection ATM network node (like a cell relay)

- An ATM network node in space

A summary of the various OBP SATM architectures is presented in Table 2. Other than the fixed ATM OBP network access architecture, the remaining architectures are still in the design/study stage. outside the radio range with a mobile user, handoffs will also occur. Issues related to user and satellite mobility, such as handoff and location management, have to be resolved. To support handoffs as a result of user or satellite mobility, both the terrestrial and satellite ATM switches have to be mobility-enabled.

Figure 16 shows the OBP SATM full mesh network architecture. The interface between a fixed ATM network and the GES is an NNI or a B ICI. If the ATM network is mobile, this interface becomes the M+NNI. For a fixed ATM user, the interface between the user station and the user terminal is the ATM UNI. However, for a mobile user this interface becomes M+UNI. With the presence of intersatellite links, the interface among satellites is the satellite NNI (S-NNI). Currently, details of S-NNI have not been finalized by TIA. Finally, an air interface is necessary between the user terminal or GES and the satellite.

The protocol reference model for OBP SATM mesh architecture shown in Fig. 17 inherits most of the earlier architectures. The RM function implemented at the GES or onboard the satellite is invoked by ATM UNI or NNI signaling messages. The RM function can optionally be implemented onboard. In this case, the GES simply transmits UNI/NNI signaling messages to the onboard ATM switch, and the RM function onboard uses the information carried in these messages to allocate/deallocate satellite capacity and channel resources. Internal signaling is used to request capacity from the NCC or release capacity based on this information. SPHY denotes the satellite physical layer, while IS-PHY denotes the intersatellite physical layer.

Mobility management functions will be implemented at the user terminals, GESs, and onboard SATM switches and ground MESs. This is necessary for the support of location management and mobile handoffs. Handoffs can be a result of mobile ATM switches in the network, mobile users, or the motion of LEO/MEO satellites, and these will be handled at the satellite and/or the MES. The NNI must be supplemented with mobility enhancements in order to support handoff-related signaling. The UNI is present at the end user to support call setup and teardown, but an $\mathrm{M}+\mathrm{UNI}$ is required to support mobile users. Since each ATM satellite is an ATM node, ATM-layer functions are present. At the physical layer, the

\section{Conclusion}

This article presents the system and network protocol architectures associated with different bent-pipe and OBP-based ATM satellites. These architectures are categorized based on types of functionality (relay, access, or interconnect), users (fixed or mobile), and network platforms (fixed or mobile). Some of these architectures are in existence, while others are still in the design stage. The shift in satellite communications paradigm from carrying conventional voice and data traffic to more time-critical and bandwidth-sensitive multimedia traffic requires new satellite architectures involving OBP ATM satellites. However, having additional components onboard the satellite can increase its cost, complexity, power consumption, and weight. Hence, such factors should be considered during the design, feasibility study, and evaluation stages. To achieve the goal of global mobile communications, global mobility, broadband access, and information transport, we envisage that the future space communications infrastructure will consist of a hybrid of GEO, MEO, and LEO space networks.

\section{Acknowledgments}

The authors would like to thank Ferit Yegenoglu, Thomas vonDeak, and the anonymous reviewers for their useful comments and constructive feedback.

\section{References}

[1] "Work fiems for Wireless ATM Access Over Geosynchronous Satellite links" ATM Forum doc. 96-1109, Aug. 1996.

[2] hitp://mww.cyberstar.com

[3] hitip://www.astrolink.com

[4] http://www. spaceway com

[5] G. Losquadro, "EUROSKYWAY: Satellite System for Interactive Multimedia Services," Proc. Ka-Band Sat. Conf. Sept. 1996.

[6] S. Agnelli and P. Mosca. "Transmission of framed ATM cells over satellite: A field experiment," Proc. IEEE ICC'95, 1995.

[7] Werner, ef al., "ATM-Based Routing in LEO/MEO Satellite Networks with Internsatellite Links," IEEE JSAC, vol. 15, no. 1, Jan. 1997.

[8] Roy Mauger and Catherine Rosenberg "QoS Guarantees for Multimedia Services on a TDMA-Based Satellite Nefwork," IEEE Commun. Mag., vol. 35, no. 7 , July 1997

[9] A. Hung et al., "A Framework for ATM via Satellite," Proc. IEEE GLOBECOM "96, Nor. 1996.

[10] K. Bhasin et al., "Satellites ATM Networks Requirements \& Architectures," Tech. draff for TIA mtg., Mar. 1997. 
[1 1] C-K. Toh ef al., "Satellite ATM Network Architectures, Protocols and Mobility Management," Tech. draff, TIA Sarellite ATM Group, June 1997.

[12] F. Yegenoglu, T. von Deak, and C-K. Toh, "Reference Models for Bend Pipe Sateilite ATM Architectures," Tech. draft submitted to ITU, Sept. 1997.

[13] I. F. Akyildiz and S-H Jeong, "Satellite ATM Networks: A Survey," IEEE Commun. Mag. vol. 35, no. 7, July 1997.

[14] T. Kaltenschnee and S. Ramseier, "Impact of burst errors on ATM over satellite - Analysis and Experimental results," Proc. 10th IEE Int'l. Conf. Digital Sat. Commun., 1995

[15] COMSAT Laboratories web site; http://www.comsat.com/atm_content/ atm.html

[16] C-K. Toh, "A Hybrid Hand-off Protocol for Local Area Wireless ATM Net works," ACM MONET, vol. 1, no. 3, Dec. 1996

[17] $M$. Erculisse et al., "An ATM-based switched VSAT communication system for LAN and Isochronous Services Interconnection," COST 226 integrated Space/Terrestrial Networks Final Symp. May 1995.

[18] J. Evans et al. "The Rapidly Deployable Radio Network," To appear. IEEE JSAC, 4th qtr., 1998 .

[19] H. Uzunalioglu, W. Yen, and I. Akyildiz, "A Connection Handover Protocol for LEO Satellite ATM Networks," ACM MOBICOM'97, Sept. 1997.

[20] M. Wittig, "Large-Capacity Multimedia Satellite Systems," IEEE Commun. Mag. vol. 35, no. 7, July 1997

[21] O. Takeda ef al., "A Study on On-Board Processor for Advanced Mobile Satellite Communications," Proc. Int'l. Mobile Sat. Conf., June 1997.

[22] [22] J. Gilderson and J. Cherkaovi, "Onboard Switching for ATM via Satellite." IEEE Commun. Mag., vol. 35, no. 7, July 1997.

[23] P. Jung ef al., "Inter-Satellite Links for Personal Communications LEO Satellite Systems," 3rd Euro. Conf. Sat. Commun., Nov. 1993.

\section{Biographies}

CHAI-KEONG TOH [M] (C-K.Toh@acm.org) received his Diploma in electronics and communication engineering with a Certificate of Merit award from the Singapore Polytechnic in 1986, his B.Eng. in electronics engineering with first class honors from the University of Manchester Institute of Science and Technology (UMIST) in 1991, and his Ph.D. degree in computer science from the Computer Laboratory, King's College, University of Cambridge, England, in 1996. He founded and chaired the Mobile Special Interest Group (Mobile SIG) from 1994 to 1996. Before joining Cambridge University, he was a network specialist, R\&D engineer, and technical staff member. At Cambridge, $\mathrm{C}-\mathrm{K}$ was an Honorary Cambridge Commonwealth Trust Scholar and a King's College Cambridge Research Scholar. He authored the book Wireless ATM and Ad Hoc Networks: Protocols and Architectures (Kluwer Academic, 1996). He serves on the editorial boards of ACM Mobile Computing and Communications Review (MC2R), (EEE Nefwork, and Personal Technologies Journal (Springer Verlag). He is a member of IEE, USENIX, ACM, Sigma Xi Honor Society, New York Academy of Science, and American Association for the Advancement of Science (AAAS), and is a Fellow of the Cambridge Philosophical Society and Cambridge Commonwealth Society. He is currently an assistant professor with the School of Electrical and Computer Engineering at the Georgia Institute of Technology, Atlanta, directing the Mobile Multimedia and High Speed Networking Laboratory.

VICTOR O. K. L [F '92] (vli@ee.hku.hk) received his S.B., S.M., and Sc.D. degrees in electrical engineering and computer science from the Massachusetts Institute of Technology, Cambridge, in 1977, 1979, and 1981, respectively. He joined the University of Southern California (USC), Los Angeles, in February 1981, and became professor of electrical engineering and director of the USC Communication Sciences Institute. Since September 1997 he has been with the University of Hong Kong, where he is chair professor of information engineering at the Department of Electrical and Electronic Engineering, and managing director of Versitech Ltd., the University Company. His research interests include high-speed communication networks, personal communication networks, and distributed multimedia systems. He has published over 200 technical articles, and has lectured and consulted extensively around the world. He chaired the Computer Communications Technical Commitree of the IEEE Communications Society from 1987 to 1989 , and the Los Angeles Chapter of the IEEE Information Theory Committee from 1983 to 1985. He chaired the Steering Committee of the International Conference on Computer Communications and Networks (IC3N) from 1992 to 1997, and was general chair of the first annual IC3N, June 1992, technical program chair of IEE Personal Communication Services Symposium, June 1995, and chair of the 4th Workstiop on Computer Communication, October 1989. He has served as an editor of IEEE Network and Telecommunication Systems, guest editor of IEEE JSAC and Computer Networks and ISDN Systems, and is now serving as an editor of ACM Wireless Networks. He was a Distinguished Lecturer at the University of California at San Diego, the National Science Council of Taiwan, and the California Polytechnic Institute. He has given keynote addresses and served on the advisory boards of numerous international conferences.

\section{IEEE/IFIP $7^{\text {th }}$ International Workshop on Quality of Service IWQOS'99}

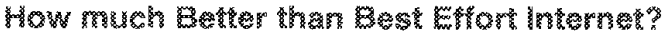

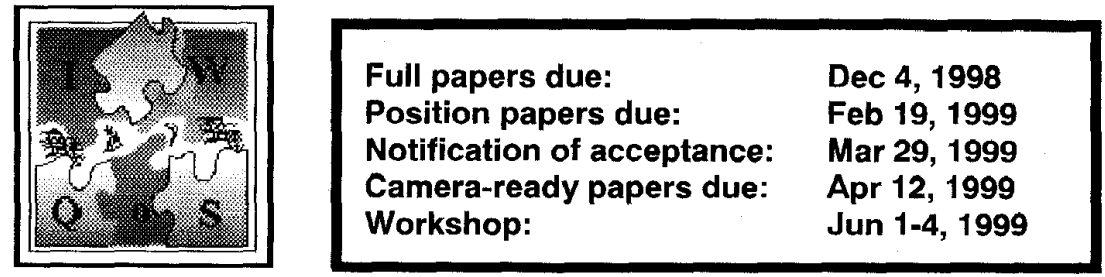

http://www.cs.ucl.ac.uk/research/iwqos99

The objective of the IWQoS'99 is to bring together researchers, developers, and practitioners working in all these areas to discuss recent innovative results and future directions. Contributions are solicited in all areas of QoS research in distributed systems and networking, including, but not limited to:

\section{QoS Architectures • Early Results with Differentiated Internet • Beyond RSVP}

\section{Multicast QoS $\downarrow$ Secure QoS $\downarrow$ Pricing $\downarrow$ Active Nets and QoS $\downarrow$ User Level QoS}

\section{Adaptive Applications $\bullet$ Terabit Forwarding and QoS $\bullet$ Routing and QoS}

\section{QoS to the Desk, Set and Home Top Mobile QoS}

In the past the workshop has been cross-disciplinary, small and well focused, consisting of keynote speakers and solicited contributions with the emphasis on innovation. As a result, a considerable amount of time is devoted to informal discussion. IWQOS'99 will be limited to 150 participants. 